\title{
On the Degradation of PtNi nanocatalysts for PEM Fuel Cells: An Identical Location Aberration-corrected STEM Study
}

\author{
Somaye Rasouli ${ }^{1}$, Tsuyohiko Fujigaya ${ }^{2}$, Deborah Myers $^{3}$, Naotoshi Nakashima ${ }^{2}$ Paulo Ferreira ${ }^{1}$ \\ 1. Materials Science and Engineering Program, University of Texas at Austin, Austin TX, USA \\ 2. Department of Applied Chemistry, Kyushu University, Fukuoka 819, Japan \\ 3. Argonne National Laboratories, Lemont, IL, USA
}

A thorough knowledge of the atomic structure and composition of electrocatalyst nanoparticles is paramount to the development of advanced materials for proton exchange membrane fuel cells (PEMFC), one of the most promising energy conversion devices for automotive and stationary applications. Pt nanoparticles (NPs) are currently used as the catalyst to promote the kinetics of the hydrogen oxidation and oxygen reduction reactions in the anode and cathode of the fuel cell, respectively. However, Pt-based alloys are being investigated to replace Pt on the cathode as a way to improve the efficiency of the fuel cell, and reduce cost [1]. Although the enhancement in the ORR activity of Pt alloys is well established, the durability of the catalysts remains the main issue for their commercialization.

In this context, the focus of this work is to understand the behavior of Pt-Ni nanoparticles during the various stages of fuel cell cycling. For this purpose a set-up was developed to simulate the effect of voltage cycling on the cathode side of the fuel cell. In this set up, Pt-Ni catalyst NPs supported on carbon were deposited on a gold TEM grid attached to a gold plate, which is used as a working electrode in a three electrode electrochemical cell. The NPs were cycled between 0.6 and $1.0 \mathrm{~V}$ in a $\mathrm{N}_{2}$ saturated $0.1 \mathrm{M} \mathrm{HClO}_{4}$ liquid electrolyte. In this fashion, pre-defined locations of the electrocatalyst on the TEM grid were analyzed before and after cycling using an aberration-corrected JEOL ARM 200F.

Fig. 1a shows that single atoms and atomic clusters appeared on the surface of the carbon support after voltage cycling as a result of surface dissolution of nanoparticles. As a result, single atoms move on the surface of the carbon support and redeposit on the surface of larger particles through modified Ostwald ripening [2]. Fig. 1b shows the surface re-deposition of single atoms on a (111) plane of a large particle. In some cases, a NP exhibits both dissolution and re-deposition at the same time. This is illustrated in Fig. 2, where atoms on surface steps are dissolved during voltage cycling, while new (111) facets are formed on the surface of the NP.

These experiments also showed the heterogeneous deposition of Pt on NPs, as shown in Figures 3a and $3 \mathrm{~b}$ (red squares 1 and 2). Initially, as there are no other particles nearby, the deposition of single atoms should have occurred, instead of coalescence. This heterogeneous deposition is likely due to the type of available surfaces in each NP. A high magnification image of the particle depicted in square 2 (Fig. 3b) is shown in Fig 3c. EDS mapping of this particle (Fig. 3d) confirms that the atomic redeposition that occurred consisted of $\mathrm{Pt}$ and not $\mathrm{Ni}$. Due to the low $\mathrm{Ni} / \mathrm{Ni}^{2+}$ redox potential and low enthalpy of mixing of $\mathrm{Pt}-\mathrm{Ni}$, re-deposition of $\mathrm{Ni}$ on the particles is not thermodynamically favorable.

References:

[1] S. C. Ball et al. ECS Transactions, 11(1) (2007), p.1267.

[2] S. Chen, et al. J. Phy. Chem. C. 113(3) (2009), p.1109. 


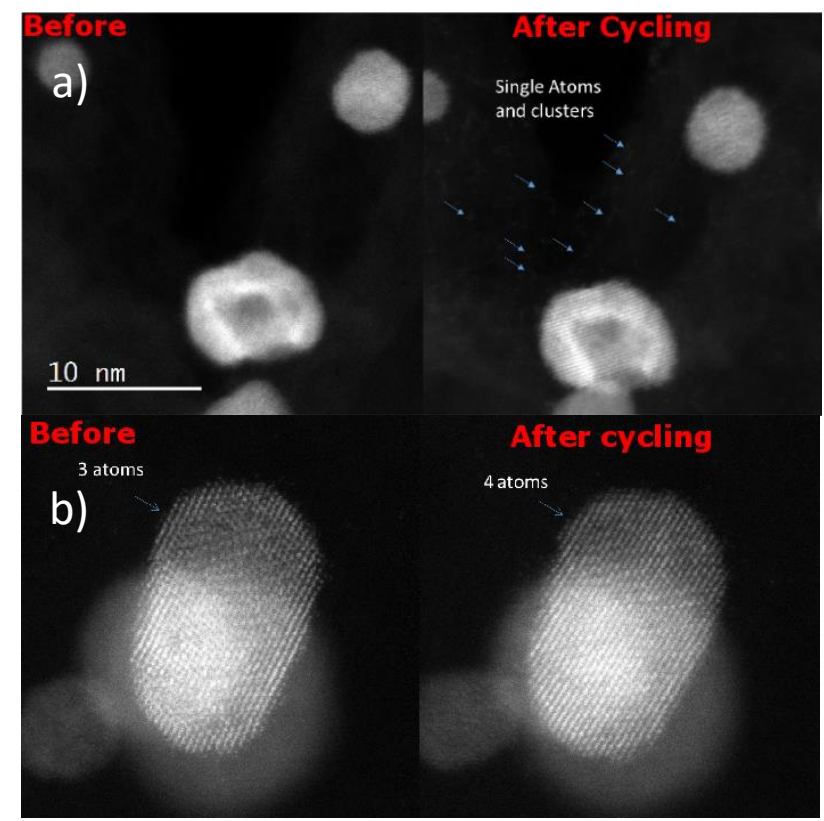

Fig. 1. a) Appearance of single atoms and atomic clusters on the carbon support after voltage cycling, b) Deposition of a single atom on the surface of a nanoparticle.
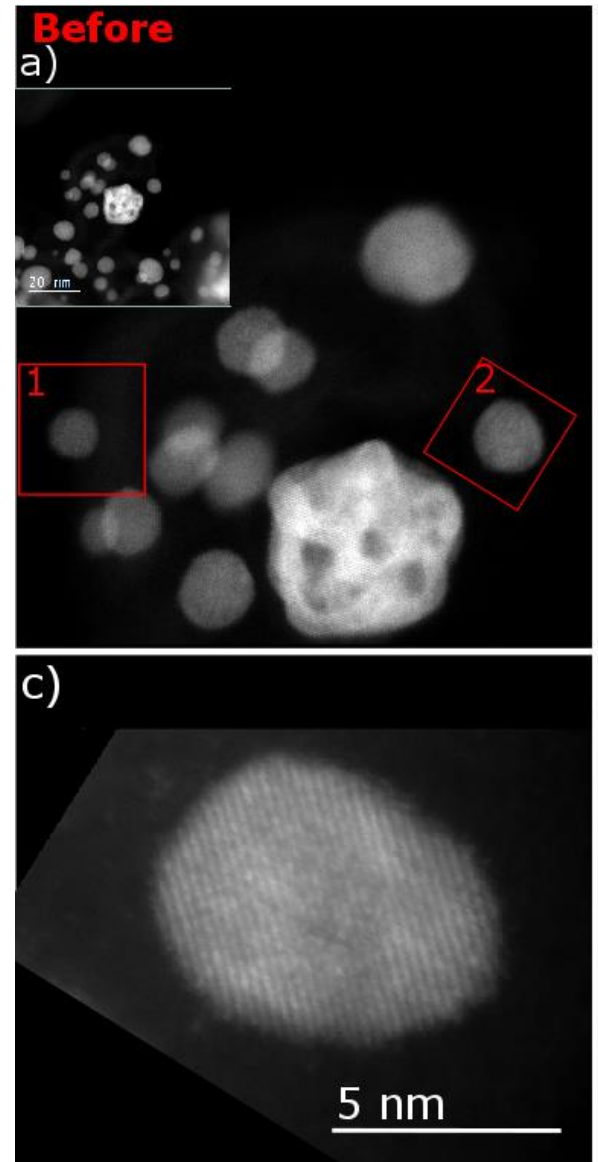

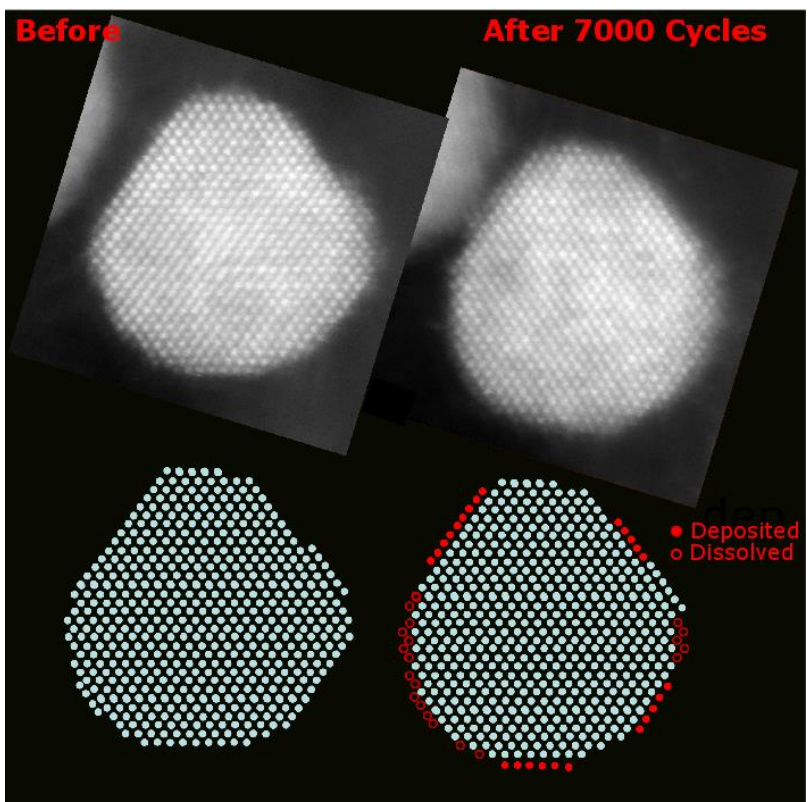

Fig. 2. Dissolution and re-deposition of surface atomic layers before and after cycling
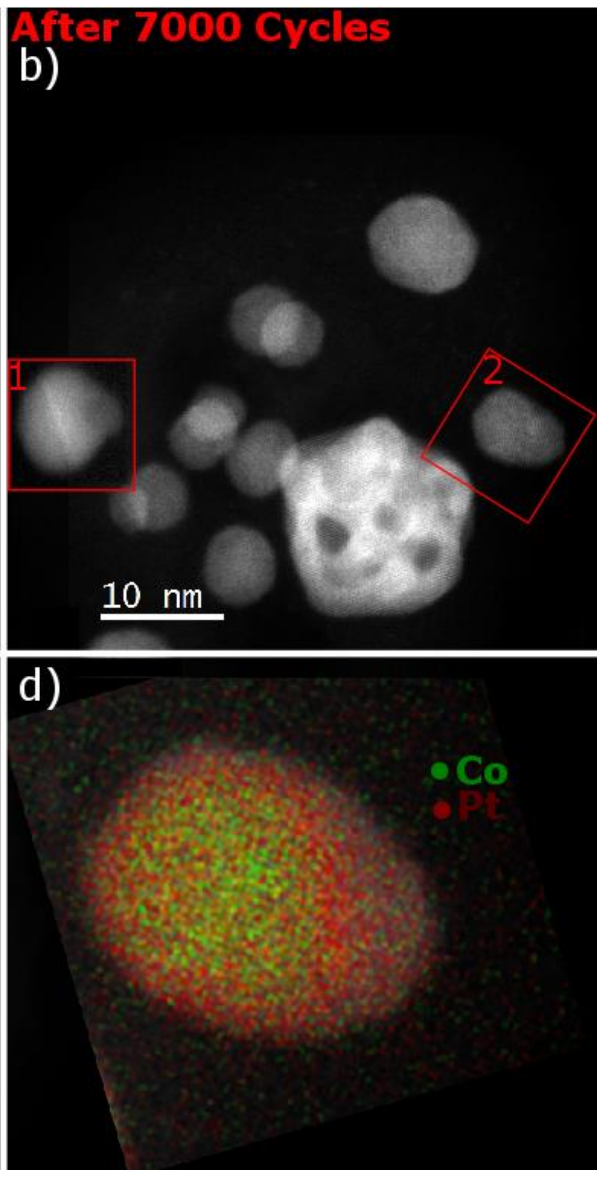

Fig. 3. Aberration corrected HAADF images of PtNi nanoparticle a) before and b) after 7000 voltage cycles, c) and d), HAADF image and EDS mapping of the PtNi nanoparticle after 7000 voltage cycles 